\title{
NEUROCOGNITIVE IMPAIRMENT (NCI) IN EUTHYMIC BIPOLAR PATIENTS: COMPARISON TO SCHIZOPHRENIA AND HEALTHY CONTROLS
}

Oluyomi Esan, Taiwo Abiona, Isaac Alakeji and Arinola Esan

Department of Psychiatry

University of Ibadan, Nigeria

Presenting Author's Full Name \&Address: Dr Oluyomi ESAN, Department of Psychiatry, University of Ibadan, and

University College Hospital , Ibadan, Nigeria. E-mail address: oluyomie@yahoo.com; Tel +2348033880312

\section{Background}

Existing literature suggest that patients with schizophrenia and patients with bipolar disorder share similar neurocognitive impairment (NCI) profile but with different degrees of severity. However the prevalence, pattern, and correlates of NCI in euthymic bipolar patients have received little attention.

Objectives : To compare NCI in euthymic bipolar patients with patients with schizophrenia in remission and healthy controls (HC)

\section{Methods}

The Screen for Cognitive Impairment in Psychiatry (SCIP) was applied in this cross-sectional study, to all consecutive and consenting euthymic outpatients with bipolar I disorder (BD-I) $(n=76)$, remitted patients with schizophrenia $(n=130)$ and age and gender matched HC $(\mathrm{n}=100)$. The study was carried out at the University College Hospital, Ibadan and the General Hospital, Adeoyo, Ibadan, Nigeria between 23 February, and 10 October 2018. Within the bipolar group, we assessed the association between NCI and social and occupational functioning using Social and Occupational Functioning Assessment Scale (SOFAS).
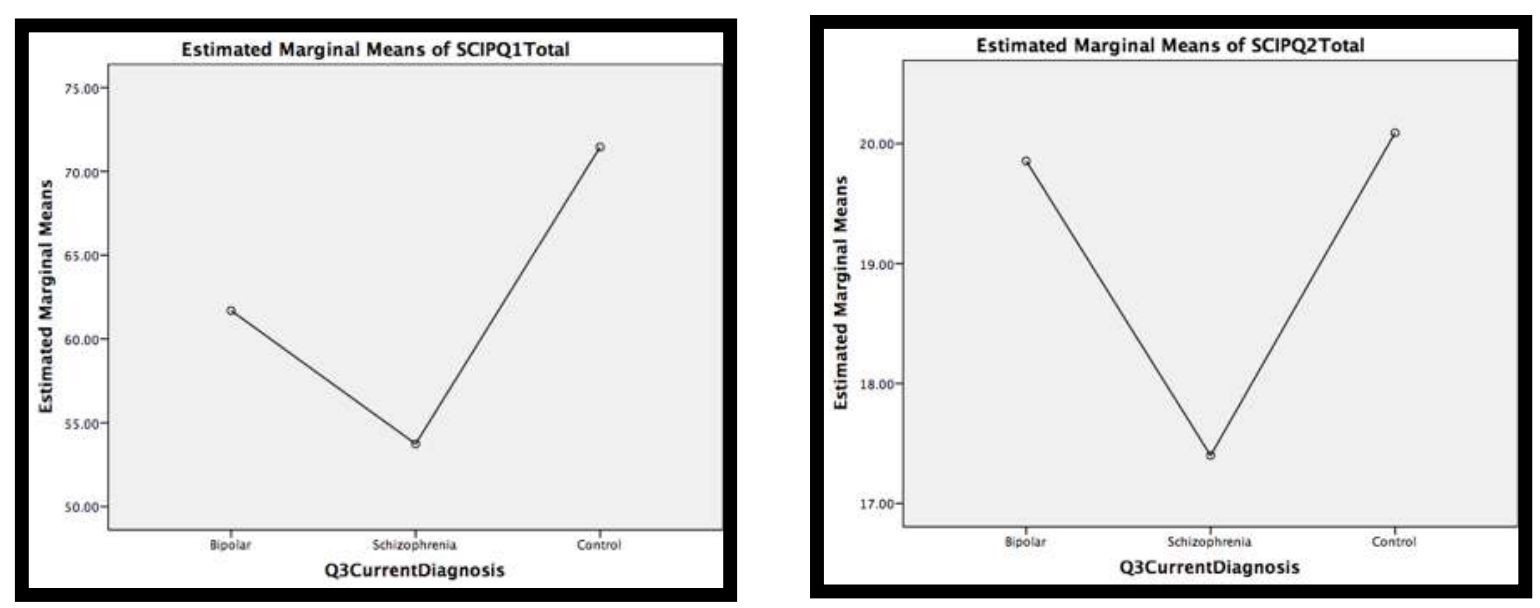

\section{Results}

The prevalence of NCI differed significantly among the three groups (9.2\%(BD-I ) vs 19.2\%(schizophrenia) vs 7.0\% (HC); $\mathrm{P}=0.013$ ). BD-I patients did significantly better than schizophrenia on verbal list learning, working memory, verbal fluency and visuomotor tracking; but poorer than HC on all components of the SCIP except working memory. Social and occupational functioning was moderately correlated with Verbal List Learning $(\mathrm{CC}=0.34 ; \mathrm{P}=0.002)$ and Visuomotor

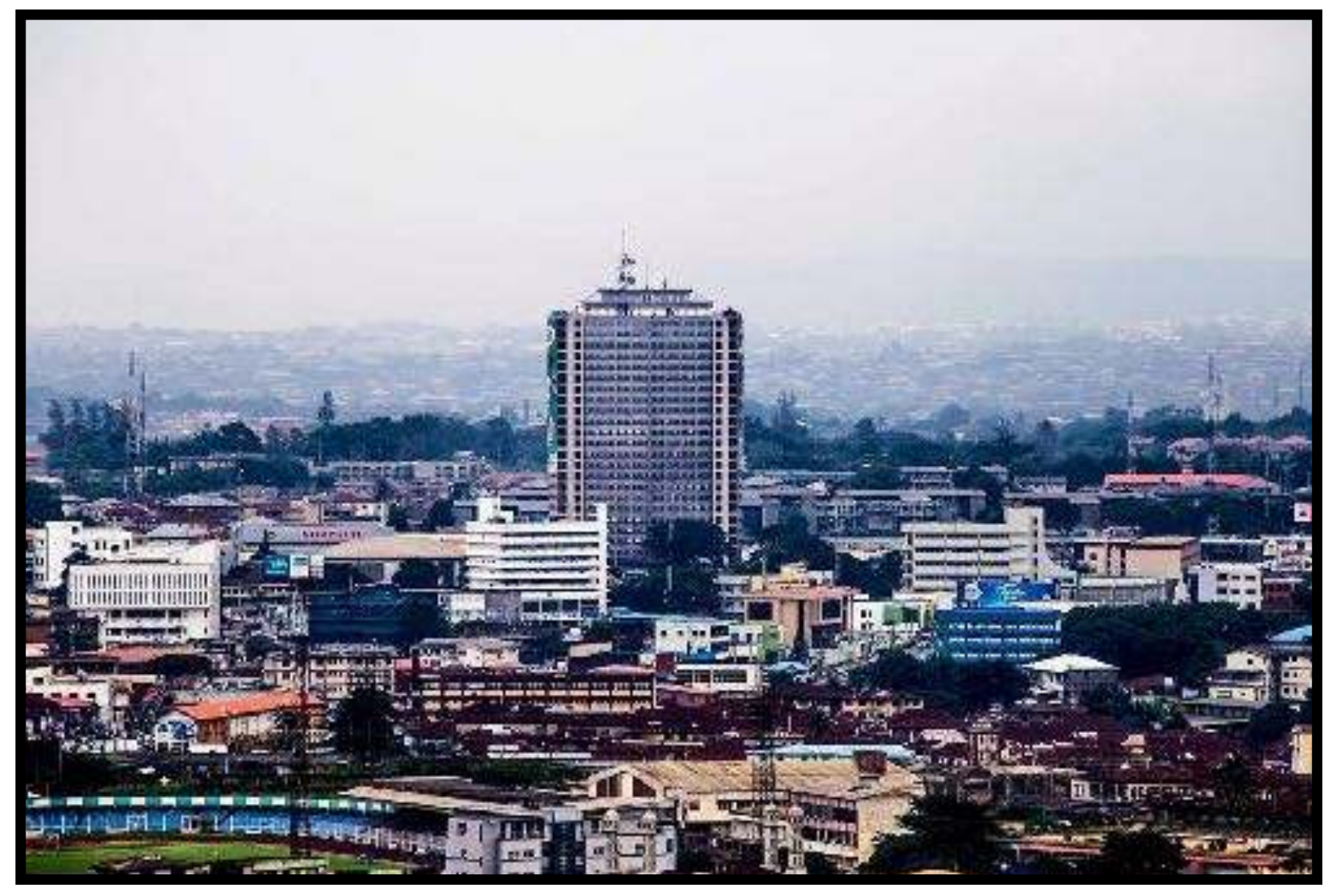

\section{Conclusion}

Despite being in a euthymic state, bipolar patients experience substantial neurocognitive impairment, This is more severe and widespread than for healthy controls. This NCI in BD-I is related to the social and occupational functioning.
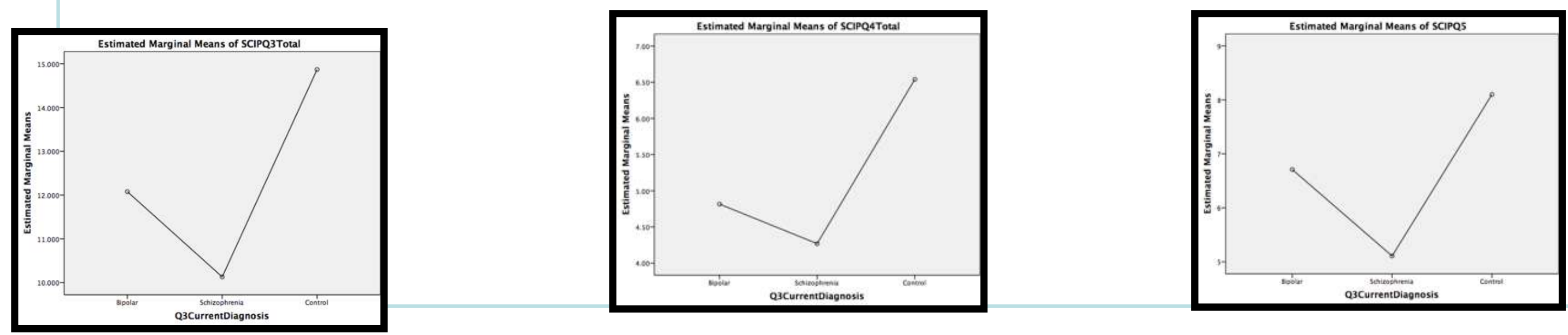

References : Depp CA, Moore DJ, Sitzer D, Palmer BW, Eyler LT, Roesch S, Lebowitz BD, Jeste DV. (2007).Neurocognitive impairment in middle-aged and older adults with bipolar disorder: comparison to schizophrenia and normal comparison subjects. J Affect Disord. 101(1-3):201-9. 